\title{
An Efficient Collocation Method for a Class of Boundary Value Problems Arising in Mathematical Physics and Geometry
}

\author{
A. H. Bhrawy, ${ }^{1,2}$ A. S. Alofi, ${ }^{1}$ and R. A. Van Gorder ${ }^{3}$ \\ ${ }^{1}$ Department of Mathematics, Faculty of Science, King Abdulaziz University, Jeddah 21589, Saudi Arabia \\ ${ }^{2}$ Department of Mathematics, Faculty of Science, Beni-Suef University, Beni-Suef 62511, Egypt \\ ${ }^{3}$ Department of Mathematics, University of Central Florida, Orlando, FL 32816, USA
}

Correspondence should be addressed to A. H. Bhrawy; alibhrawy@yahoo.co.uk

Received 24 March 2014; Revised 2 May 2014; Accepted 3 May 2014; Published 15 May 2014

Academic Editor: Dumitru Baleanu

Copyright (C) 2014 A. H. Bhrawy et al. This is an open access article distributed under the Creative Commons Attribution License, which permits unrestricted use, distribution, and reproduction in any medium, provided the original work is properly cited.

\begin{abstract}
We present a numerical method for a class of boundary value problems on the unit interval which feature a type of powerlaw nonlinearity. In order to numerically solve this type of nonlinear boundary value problems, we construct a kind of spectral collocation method. The spatial approximation is based on shifted Jacobi polynomials $J_{n}^{(\alpha, \beta)}(r)$ with $\alpha, \beta \in(-1, \infty), r \in(0,1)$ and $n$ the polynomial degree. The shifted Jacobi-Gauss points are used as collocation nodes for the spectral method. After deriving the method for a rather general class of equations, we apply it to several specific examples. One natural example is a nonlinear boundary value problem related to the Yamabe problem which arises in mathematical physics and geometry. A number of specific numerical experiments demonstrate the accuracy and the efficiency of the spectral method. We discuss the extension of the method to account for more complicated forms of nonlinearity.
\end{abstract}

\section{Introduction}

Spectral methods (see, for instance, [1-6]) are one of the principal methods of discretization for the numerical solution of differential equations. The three most widely used spectral versions are the Galerkin, collocation, and tau methods. Collocation methods [7-12] have become increasingly popular for solving differential equations, also they are very useful in providing highly accurate solutions to nonlinear differential equations. In the present paper, we intend to extend the application of Jacobi polynomials from Galerkin method for solving two-point linear problems (see $[13,14]$ ) to collocation method to solve nonlinear second-order initial value problems. To the best of our knowledge, there are not so many results on Jacobi-Gauss collocation method for differential equations of second-order arising in mathematical physics. This partially motivated our interest in such a method.

A well-known advantage of a spectral method is that it achieves high accuracy with relatively fewer spatial grid points when compared with a finite-difference method. On the other hand, spectral methods typically give rise to full matrices, partially negating the gain in efficiency due to the fewer number of grid points. In general, the use of Jacobi polynomials $\left(P_{k}^{(\alpha, \beta)}\right.$ with $\alpha, \beta \in(-1, \infty)$ and $n$ is the polynomial degree) has the advantage of obtaining the solutions of differential equations in terms of the Jacobi indexes $\alpha$ and $\beta$ (see, e.g., $[15,16]$ ).

Each of these particular pairs of $\alpha$ and $\beta$ has been used separately for solving approximately differential equations (see [17-20]). Hence, to generalize and instead of developing approximation results for each particular pair of indexes, it would be very useful to carry out a systematic study on Jacobi polynomials with general indexes which can then be directly applied to other applications [21].

In the present paper, we will consider the nonlinear ordinary boundary value problem

$$
\begin{aligned}
& v^{\prime \prime}+\frac{1}{r} v^{\prime}-v+\lambda v^{n}=0, \\
& v^{\prime}(0)=0, \quad v(1)=1 .
\end{aligned}
$$


Here $\lambda$ is a nonzero parameter, while $n$ is the power-law index. The larger the deviation of $n$ from either 1 or 0 , the stronger the nonlinearity. Physically, it makes sense to consider $n>0$. Note that the final boundary condition is sufficiently general. Indeed, if we are given $\nu(a)=b$, we may always rescale the dependent and independent variables to get $v(1)=1$ provided that $a \neq 0$ and $b \neq 0$.

One example of such a boundary value problem would be for the Yamabe equation on a sphere. The Yamabe equation is a nonlinear differential equation arising in geometry and related areas of mathematical physics [22-24]. Under the assumption of radial solutions, the Yamabe problem can be put into correspondence with (1)-(2) for appropriate $n$ and $\lambda$. Previously, some approximate analytical results were obtained by Van Gorder [25].

Note also that the boundary value problem somewhat resembles the Lane-Emden problem of the first kind [2629 ], since there is a power-law nonlinearity. However, the Lane-Emden problem of the first kind is different in two ways. First, the linear term $-v$ is not present in the LaneEmden problem. This term alters the linearized eigenvalue problem and hence would change the base functions used in any approximation scheme. Secondly, the present problem is a boundary value problem, while the Lane-Emden problem of the first kind is an initial value problem with initial conditions $\nu(0)=1, v^{\prime}(0)=0$. So, the present problem is rather different from those previously considered in the literature and hence merits study. Another difference is that, in the Lane-Emden problem of the first kind, the power-law index is often an integer. Here, the value of $n$ is any positive rational number. As it turns out, $n$ is often not an integer, in the case of problems such as the Yamabe problem.

The fundamental goal of this paper is to develop a suitable way to approximate power-law nonlinear ODEs (1) with boundary conditions $(2)$ on the interval $(0,1)$ numerically using the Jacobi polynomials. We propose a spectral shifted Jacobi-Gauss collocation (SJC) method to find an approximate numerical solution $v_{N}(r)$. The ODE (1) is collocated only at $(N-1)$ points. For suitable collocation points we use the $N-1$ nodes of the shifted Jacobi-Gauss interpolation on $(0,1)$. These equations together with initial condition generate $(N+1)$ algebraic equations which can be solved using Newton's iterative method. Finally, the accuracy of the proposed methods is demonstrated by test problems, and numerical results are presented in which the exponential convergence behavior of spectral approximations is exhibited. The numerical experiments demonstrate that the method is both efficient and accurate.

This paper is organized as follows. In Section 2 we give an overview of shifted Jacobi polynomials and their relevant properties needed hereafter. Then, in Section 3, we construct the collocation method for (1) using the shifted Jacobi polynomials. We derive the numerical method for general values of the model parameters $\lambda$ and $n$. In Section 4 , we present some numerical results exhibiting the accuracy and efficiency of our numerical algorithms. We consider a wide variety of parameter regimes, in order to demonstrate the robustness of the numerical scheme. Such results are related to radial solutions of the Yamabe problem. In particular, in the Yamabe problem the power-law index $n$ is a function of the spatial dimension, and as the spatial dimension increases, the power-law index remains bounded like $1<n \leq 5$. Hence, we are able to use the present results to infer properties of solutions to the Yamabe problem.

\section{Preliminaries}

Let $\alpha>-1, \beta>-1$, and $P_{k}^{(\alpha, \beta)}(r)$ be the standard Jacobi polynomial of degree $k$. Then we have that

$$
\begin{gathered}
P_{k}^{(\alpha, \beta)}(-r)=(-1)^{k} P_{k}^{(\alpha, \beta)}(r), \\
P_{k}^{(\alpha, \beta)}(-1)=\frac{(-1)^{k} \Gamma(k+\beta+1)}{k ! \Gamma(\beta+1)}, \\
P_{k}^{(\alpha, \beta)}(1)=\frac{\Gamma(k+\alpha+1)}{k ! \Gamma(\alpha+1)} .
\end{gathered}
$$

Furthermore, note that

$$
D^{m} P_{k}^{(\alpha, \beta)}(r)=2^{-m} \frac{\Gamma(m+k+\alpha+\beta+1)}{\Gamma(k+\alpha+\beta+1)} P_{k-m}^{(\alpha+m, \beta+m)}(r) .
$$

Let $w^{(\alpha, \beta)}(r)=(1-r)^{\alpha}(1+r)^{\beta}$. We define the weighted space $L_{w^{(\alpha, \beta)}}^{2}[-1,1]$ as usual, equipped with the following inner product and norm:

$$
\begin{gathered}
(u, v)_{w^{(\alpha, \beta)}}=\int_{-1}^{1} u(r) v(r) w^{(\alpha, \beta)}(r) d x, \\
\|v\|_{w^{(\alpha, \beta)}}=(v, v)_{w^{(\alpha, \beta)}}^{1 / 2} .
\end{gathered}
$$

The set of Jacobi polynomials forms a complete $L_{w^{\alpha, \beta}}^{2}[-1,1]$ orthogonal system, and

$$
\begin{aligned}
\left\|P_{k}^{(\alpha, \beta)}\right\|_{w^{(\alpha, \beta)}}^{2} & =h_{k}^{(\alpha, \beta)} \\
& =\frac{2^{\alpha+\beta+1} \Gamma(k+\alpha+1) \Gamma(k+\beta+1)}{(2 k+\alpha+\beta+1) \Gamma(k+1) \Gamma(k+\alpha+\beta+1)} .
\end{aligned}
$$

If we define the shifted Jacobi polynomial of degree $k$ by $J_{k}^{(\alpha, \beta)}(r)=P_{k}^{(\alpha, \beta)}(2 r-1)$, and in virtue of (3) and (4), then it can be easily shown that

$$
\begin{gathered}
D^{q} J_{k}^{(\alpha, \beta)}(0)=\frac{(-1)^{k-q} \Gamma(k+\beta+1)(k+\alpha+\beta+1)_{q}}{\Gamma(k-q+1) \Gamma(q+\beta+1)}, \\
D^{q} J_{k}^{(\alpha, \beta)}(1)=\frac{\Gamma(k+\alpha+1)(k+\alpha+\beta+1)_{q}}{\Gamma(k-q+1) \Gamma(q+\alpha+1)}, \\
D^{m} J_{k}^{(\alpha, \beta)}(r)=\frac{\Gamma(m+k+\alpha+\beta+1)}{\Gamma(k+\alpha+\beta+1)} J_{k-m}^{(\alpha+m, \beta+m)}(r) .
\end{gathered}
$$


Next, let $\chi^{(\alpha, \beta)}(r)=(1-r)^{\alpha} r^{\beta}$. We define the weighted space $L_{\chi^{(\alpha, \beta)}}^{2}[0,1]$ in the usual way, with the following inner product and norm:

$$
\begin{gathered}
(u, v)_{\chi^{(\alpha, \beta)}}=\int_{0}^{1} u(r) v(r) \chi^{(\alpha, \beta)}(r) d x, \\
\|v\|_{\chi^{(\alpha, \beta)}}=(v, v)_{\chi^{(\alpha, \beta)}}^{1 / 2} .
\end{gathered}
$$

The set of shifted Jacobi polynomials forms a complete $L_{\chi^{(\alpha, \beta)}}^{2}[0,1]$-orthogonal system. Moreover, and due to $(6)$, we have

$$
\left\|J_{k}^{(\alpha, \beta)}\right\|_{\chi^{(\alpha, \beta)}}^{2}=\left(\frac{1}{2}\right)^{\alpha+\beta+1} h_{k}^{(\alpha, \beta)}=\eta_{k}^{(\alpha, \beta)} .
$$

For $\alpha=\beta$ one recovers the shifted ultraspherical polynomials (symmetric Jacobi polynomials) and for $\alpha=\beta=\mp 1 / 2, \alpha=$ $\beta=0$ we recover the shifted Chebyshev of the first and second kinds and the shifted Legendre polynomials, respectively. For the nonsymmetric shifted Jacobi polynomials, the two important special cases $\alpha=-\beta= \pm 1 / 2$ (shifted Chebyshev polynomials of the third and fourth kinds) are also recovered.

We denote by $r_{N, j}^{(\alpha, \beta)}, 0 \leqslant j \leqslant N$ the nodes of the standard Jacobi-Gauss interpolation on the interval $[-1,1]$. Their corresponding Christoffel numbers are $\omega_{N, j}^{(\alpha, \beta)}, 0 \leqslant j \leqslant$ $N$. The nodes of the shifted Jacobi-Gauss interpolation on the interval $[0,1]$ are the zeros of $J_{N+1}^{(\alpha, \beta)}(r)$, which we denote by $\theta_{N, j}^{(\alpha, \beta)}, 0 \leqslant j \leqslant N$. Clearly $\theta_{N, j}^{(\alpha, \beta)}=(1 / 2)\left(r_{N, j}^{(\alpha, \beta)}+1\right)$ and their corresponding Christoffel numbers are $\vartheta_{N, j}^{(\alpha, \beta)}=$ $(1 / 2)^{\alpha+\beta+1} \omega_{N, j}^{(\alpha, \beta)}, 0 \leqslant j \leqslant N$. Let $S_{N}[0,1]$ be the set of polynomials of degree at most $N$. Thanks to the property of the standard Jacobi-Gauss quadrature, it follows that for any $\phi \in S_{2 N+1}[0,1]$,

$$
\begin{aligned}
\int_{0}^{1}(1 & -r)^{\alpha} r^{\beta} \phi(x) d x \\
& =\left(\frac{1}{2}\right)^{\alpha+\beta+1} \int_{-1}^{1}(1-r)^{\alpha}(1+r)^{\beta} \phi\left(\frac{1}{2}(r+1)\right) d r \\
& =\left(\frac{1}{2}\right)^{\alpha+\beta+1} \sum_{j=0}^{N} \omega_{N, j}^{(\alpha, \beta)} \phi\left(\frac{1}{2}\left(r_{N, j}^{(\alpha, \beta)}+1\right)\right) \\
& =\sum_{j=0}^{N} \vartheta_{N, j}^{(\alpha, \beta)} \phi\left(\theta_{N, j}^{(\alpha, \beta)}\right) .
\end{aligned}
$$

\section{Shifted Jacobi-Gauss Collocation Method}

In this section, we consider a Jacobi-Gauss collocation method approach to numerically solve the nonlinear boundary value problem

$$
\nu^{\prime \prime}(r)+\frac{1}{r} \nu^{\prime}(r)-\nu(r)+\lambda \nu^{n}(r)=0
$$

subject to

$$
\nu^{\prime}(0)=0, \quad \nu(1)=1 .
$$

The choice of collocation points is important for the convergence and efficiency of the collocation method. It should be noted that for a second-order differential equation with the singularity at $r=0$ in the interval $(0,1)$, one is unable to apply the collocation method with Jacobi-GaussRadau points because the fixed node $r=0$ is necessary to use as a point from the collocation nodes. In fact, the collocation method with Jacobi-Gauss nodes are used to treat singular second-order differential equation; that is, we collocate the singular nonlinear ODE only at the $(N-1)$ Jacobi-Gauss points that are the $(N-1)$ zeros of the shifted Jacobi polynomial on $(0,1)$. These equations together with two initial conditions generate $(N+1)$ nonlinear algebraic equations which can be solved.

Let us first introduce some basic notation. We set

$$
S_{N}(0,1)=\operatorname{span}\left\{J_{0}^{(\alpha, \beta)}(r), J_{1}^{(\alpha, \beta)}(r), \ldots, J_{N}^{(\alpha, \beta)}(r)\right\}
$$

and we define the discrete inner product and norm as follows:

$$
\begin{gathered}
(u, v)_{\chi^{(\alpha, \beta), N}}=\sum_{j=0}^{N} u\left(\theta_{N, j}^{(\alpha, \beta)}\right) v\left(\theta_{N, j}^{(\alpha, \beta)}\right) \vartheta_{N, j}^{(\alpha, \beta)}, \\
\|u\|_{\chi^{(\alpha, \beta), N}}=\sqrt{(u, u)_{\chi^{(\alpha, \beta)}, N}} .
\end{gathered}
$$

Here $\theta_{N, j}^{(\alpha, \beta)}$ and $\vartheta_{N, j}^{(\alpha, \beta)}$ are the nodes and the corresponding weights of the shifted Jacobi-Gauss quadrature formula on the interval $(0,1)$, respectively. Obviously,

$$
(u, v)_{\chi^{(\alpha, \beta)}, N}=(u, v)_{\chi^{(\alpha, \beta)}}, \quad \forall u, v \in S_{2 N-1} .
$$

Thus, for any $u \in S_{N}(0,1)$, the norms $\|u\|_{\chi^{(\alpha, \beta)}, N}$ and $\|u\|_{\chi^{(\alpha, \beta)}}$ coincide.

Associating with this quadrature rule, we denote by $I_{N}^{J^{(\alpha, \beta)}}$ the shifted Jacobi-Gauss interpolation,

$$
I_{N}^{J^{(\alpha, \beta)}} u\left(\theta_{N, j}^{(\alpha, \beta)}\right)=u\left(\theta_{N, j}^{(\alpha, \beta)}\right), \quad 0 \leq k \leq N .
$$

The shifted Jacobi-Gauss collocation method for solving (13) and (14) is to seek $\nu_{N}(x) \in S_{N}(0,1)$, such that

$$
\begin{array}{r}
\nu^{\prime \prime}\left(\theta_{N, k}^{(\alpha, \beta)}\right)+\frac{1}{\theta_{N, k}^{(\alpha, \beta)}} \nu^{\prime}\left(\theta_{N, k}^{(\alpha, \beta)}\right)-\nu\left(\theta_{N, k}^{(\alpha, \beta)}\right)+\lambda \nu^{n}\left(\theta_{N, k}^{(\alpha, \beta)}\right)=0, \\
k=0,1, \ldots, N-2 . \\
v_{N}^{\prime}(0)=0, \quad v_{N}(1)=1 .
\end{array}
$$

We now derive an algorithm for solving (13) and (14). To do this, let

$$
\nu_{N}(r)=\sum_{j=0}^{N} a_{j} J_{j}^{(\alpha, \beta)}(r), \quad a=\left(a_{0}, a_{1}, \ldots, a_{N}\right)^{T} .
$$


We first approximate $v(r), \nu^{\prime}(r)$, and $\nu^{\prime \prime}(r)$, as (20). By substituting these approximation in (13), we get

$$
\begin{aligned}
\sum_{j=0}^{N} a_{j} D^{2} J_{j}^{(\alpha, \beta)}(r) & \frac{1}{r} \sum_{j=0}^{N} a_{j} D J_{j}^{(\alpha, \beta)}(r) \\
& -\sum_{j=0}^{N} a_{j} J_{j}^{(\alpha, \beta)}(r)+\lambda\left(\sum_{j=0}^{N} a_{j} J_{j}^{(\alpha, \beta)}(r)\right)^{n}=0 .
\end{aligned}
$$

Then, by virtue of (9), we deduce that

$$
\begin{aligned}
\sum_{j=0}^{N} a_{j}(j+\alpha+\beta+1)_{2} J_{j-2}^{(\alpha+2, \beta+2)}(r) & \\
+ & \frac{1}{r} \sum_{j=0}^{N} a_{j}(j+\alpha+\beta+1) J_{j-1}^{(\alpha+1, \beta+1)}(r) \\
& -\sum_{j=0}^{N} a_{j} J_{j}^{(\alpha, \beta)}(r)+\lambda\left(\sum_{j=0}^{N} a_{j} J_{j}^{(\alpha, \beta)}(r)\right)^{n}=0 .
\end{aligned}
$$

Also, by substituting (20) in (14) we obtain

$$
\sum_{j=0}^{N} a_{j} D J_{j}^{(\alpha, \beta)}(0)=0, \quad \sum_{j=0}^{N} a_{j} J_{j}^{(\alpha, \beta)}(1)=1 .
$$

To find the solution $v_{N}(r)$, we first collocate $(22)$ at the $(N-1)$ Jacobi rational roots, yielding

$$
\begin{aligned}
& \sum_{j=0}^{N} a_{j}(j+\alpha+\beta+1)_{2} J_{j-2}^{(\alpha+2, \beta+2)}(r) \\
& +\frac{1}{r} \sum_{j=0}^{N} a_{j}(j+\alpha+\beta+1) J_{j-1}^{(\alpha+1, \beta+1)}(r) \\
& \quad-\sum_{j=0}^{N} a_{j} J_{j}^{(\alpha, \beta)}(r)+\lambda\left(\sum_{j=0}^{N} a_{j} J_{j}^{(\alpha, \beta)}(r)\right)^{n}=0 .
\end{aligned}
$$

Equation (23), after using (7) and (8), can be written as

$$
\begin{gathered}
\sum_{j=1}^{N}(-1)^{j-1} \frac{(j+\alpha+\beta+1) \Gamma(j+\beta+1)}{(j-1) ! \Gamma(\beta+2)} a_{j}=0, \\
\sum_{j=0}^{N} \frac{\Gamma(j+\alpha+1)}{\Gamma(j-1) \Gamma(\alpha+1)} a_{j}=0 .
\end{gathered}
$$

Finally, from (24) and (25), we get $(N+1)$ nonlinear algebraic equations which can be solved for the unknown coefficients $a_{j}$ by using any standard iteration technique, like Newton's iteration method. Consequently, $v_{N}(r)$ given in (20) can be evaluated.

With this, we have derived the relevant spectral method for the boundary value problems (1)-(2). In what follows, we will apply the method to obtain approximate numerical solutions to some specific values of $\lambda$ and $n$. In doing so, we will be able to demonstrate the accuracy and efficiency of the method.

\section{Numerical Experiments and Examples}

The Yamabe equation is a nonlinear differential equation arising in geometry and related areas of mathematical physics [22-24]. Previously, some approximate analytical results were obtained by Van Gorder [25]. We will consider the Yamabe equation on the unit ball $\mathbf{B}^{m}$ in $\mathbb{R}^{m}$, where $m=3,4,5, \ldots$ is the dimension of the space. Previously, some approximate analytical results were obtained by Van Gorder [25]. However, the analytical method employed there becomes complicated after a number of iterations, hindering computational efficiency. The Yamabe equation is a strongly nonlinear partial differential equation for any choice of $m$ greater than two. Note that the nonlinearity strongly depends on the problem dimension. To simplify the situation, we assume a radially symmetric solution

$$
u(x)=v(r) .
$$

For simplicity of notation, let us also pick the constant

$$
n=1+\frac{4}{(m-2)}
$$

so that for $m \geq 3$ we have $n \in(1,5]$. Then, under appropriate scaling of the dependent and independent variables, the Yamabe is put into the form

$$
\begin{aligned}
& v^{\prime \prime}+\frac{1}{r} \nu^{\prime}-v+\lambda v^{n}=0, \\
& v^{\prime}(0)=0, \quad v(1)=1 .
\end{aligned}
$$

Here $\lambda$ is a parameter. We should remark that (28) is similar in form to the Lane-Emden equation of the first kind which has been considered in the literature [26-29]. Note that (28) has an extra term, and that the form of the conditions is different (the relevant Lane-Emden problem is an initial value problem, not a boundary value problem). So, this problem will be a bit harder to solve than the Lane-Emden initial value problem. Both $\lambda$ and $n$ are parameters. We can take $\lambda$ to be a real number, whereas $n$ depends on $m$ : if $m=3, n=5$; if $m=4, n=3$; if $m=5, n=7 / 3$; and so on. As $m$ tends to infinity, $n$ tends to 1 , so the problem is linear in this limit and the problem can be solved exactly.

In this section we show the accuracy and rapid convergence of the proposed spectral method for this boundary value problem. Table 1 shows the approximations of $v_{N}(r)$ for indices $n=5,3$ and $\lambda=1 / 2,4 / 3$ respectively, with $\alpha=\beta=-1 / 2$ (first kind shifted Chebyshev collocation method), $\alpha=\beta=0$ (shifted Legendre collocation method), and $\alpha=\beta=1 / 2$ (second kind shifted Chebyshev collocation method) at $N=24$. Moreover, we list the values of the approximate solution at $N=24$ for the two special choices $n=7 / 3,2$, and $\lambda=1 / 3,-1 / 3$ respectively in Table 2 .

Tables 3, 4, 5, and 6 show absolute residual errors of $\nu_{N}(r)$ for indices $n=5,3,7 / 3,2$, and $\lambda=1 / 2,4 / 3,1 / 3$, $-1 / 3$, respectively, with various choices of $\alpha$ and $\beta$ at $N=$ 16 and $N=24$. In these tables, we compare the results obtained by adopting different choices of the two shifted Jacobi parameters $\alpha$ and $\beta$, and we conclude that the present 
TABLE 1: Approximate solutions for $N=24$.

\begin{tabular}{|c|c|c|c|c|c|c|}
\hline$r$ & $\alpha=\beta=-1 / 2$ & $\begin{array}{c}\alpha=\beta=0 \\
(n=5, \lambda=1 / 2)\end{array}$ & $\alpha=\beta=1 / 2$ & $\alpha=\beta=-1 / 2$ & $\begin{array}{c}\alpha=\beta=0 \\
(n=3, \lambda=4 / 3)\end{array}$ & $\alpha=\beta=1 / 2$ \\
\hline 0.0 & 0.847890 & 0.847890 & 0.847890 & 1.26779 & 1.26779 & 1.26779 \\
\hline 0.1 & 0.849462 & 0.849462 & 0.849462 & 1.26418 & 1.26418 & 1.26418 \\
\hline 0.2 & 0.854173 & 0.854173 & 0.854173 & 1.125350 & 1.125350 & 1.125350 \\
\hline 0.3 & 0.862013 & 0.862013 & 0.862013 & 1.23616 & 1.23616 & 1.23616 \\
\hline 0.4 & 0.872959 & 0.872959 & 0.872959 & 1.21283 & 1.21283 & 1.21283 \\
\hline 0.5 & 0.886976 & 0.886976 & 0.886976 & 1.18437 & 1.18437 & 1.18437 \\
\hline 0.6 & 0.904009 & 0.904009 & 0.904009 & 1.15174 & 1.15174 & 1.15174 \\
\hline 0.7 & 0.923974 & 0.923974 & 0.923974 & 1.11599 & 1.11599 & 1.11599 \\
\hline 0.8 & 0.946751 & 0.946751 & 0.946751 & 1.07815 & 1.07815 & 1.07815 \\
\hline 0.9 & 0.972170 & 0.972170 & 0.972170 & 1.03918 & 1.03918 & 1.03918 \\
\hline 1.0 & 1.000000 & 1.000000 & 1.000000 & 1.00000 & 1.00000 & 1.00000 \\
\hline
\end{tabular}

TABLE 2: Approximate solutions for $N=24$.

\begin{tabular}{|c|c|c|c|c|c|c|}
\hline$r$ & $\alpha=\beta=-1 / 2$ & $\begin{array}{c}\alpha=\beta=0 \\
(n=7 / 3, \lambda=1 / 3)\end{array}$ & $\alpha=\beta=1 / 2$ & $\alpha=\beta=-1 / 2$ & $\begin{array}{c}\alpha=\beta=0 \\
(n=2, \lambda=-1 / 3)\end{array}$ & $\alpha=\beta=1 / 2$ \\
\hline 0.0 & 0.841907 & 0.841907 & 0.841907 & 0.744476 & 0.744476 & 0.744476 \\
\hline 0.1 & 0.843455 & 0.843455 & 0.843455 & 0.746801 & 0.746801 & 0.746801 \\
\hline 0.2 & 0.848101 & 0.848101 & 0.848101 & 0.753803 & 0.753803 & 0.753803 \\
\hline 0.3 & 0.855860 & 0.855860 & 0.855860 & 0.765560 & 0.765560 & 0.765560 \\
\hline 0.4 & 0.866753 & 0.866753 & 0.866753 & 0.782206 & 0.782206 & 0.782206 \\
\hline 0.5 & 0.880809 & 0.880809 & 0.880809 & 0.803932 & 0.803932 & 0.803932 \\
\hline 0.6 & 0.898065 & 0.898065 & 0.898065 & 0.830988 & 0.830988 & 0.830988 \\
\hline 0.7 & 0.918563 & 0.918563 & 0.918563 & 0.863692 & 0.863692 & 0.863692 \\
\hline 0.8 & 0.942351 & 0.942351 & 0.942351 & 0.902433 & 0.902433 & 0.902433 \\
\hline 0.9 & 0.969479 & 0.969479 & 0.969479 & 0.947681 & 0.947681 & 0.947681 \\
\hline 1.0 & 1.000000 & 1.000000 & 1.000000 & 1.000000 & 1.000000 & 1.000000 \\
\hline
\end{tabular}

TABLE 3: Absolute residual error functions for $n=5, \lambda=1 / 2$.

\begin{tabular}{llllccc}
\hline$r$ & $\alpha=\beta=-1 / 2$ & $\begin{array}{l}\alpha=\beta=0 \\
(N=16)\end{array}$ & $\alpha=\beta=1 / 2$ & $\alpha=\beta=-1 / 2$ & $\begin{array}{l}\alpha=\beta=0 \\
(N=24)\end{array}$ \\
\hline 0.0 & $1.16 \cdot 10^{-14}$ & $6.29 \cdot 10^{-15}$ & $2.69 \cdot 10^{-14}$ & $7.45 \cdot 10^{-15}$ & $8.97 \cdot 10^{-15}$ \\
0.1 & $3.66 \cdot 10^{-15}$ & $4.66 \cdot 10^{-15}$ & $3.88 \cdot 10^{-15}$ & $1.11 \cdot 10^{-16}$ & $4.44 \cdot 10^{-16}$ \\
0.2 & $4.88 \cdot 10^{-15}$ & $1.88 \cdot 10^{-15}$ & $1.11 \cdot 10^{-16}$ & $1.11 \cdot 10^{-16}$ & $1.11 \cdot 10^{-16}$ \\
0.3 & $9.54 \cdot 10^{-15}$ & $6.21 \cdot 10^{-15}$ & $4.10 \cdot 10^{-15}$ & $1.11 \cdot 10^{-16}$ & $1.11 \cdot 10^{-16}$ & 0 \\
0.4 & $1.39 \cdot 10^{-14}$ & $9.65 \cdot 10^{-15}$ & $6.43 \cdot 10^{-15}$ & 0 & $2.22 \cdot 10^{-16}$ \\
0.5 & $1.97 \cdot 10^{-14}$ & $1.33 \cdot 10^{-14}$ & $9.43 \cdot 10^{-15}$ & $1.11 \cdot 10^{-16}$ & 0 \\
0.6 & $2.93 \cdot 10^{-14}$ & $2.00 \cdot 10^{-14}$ & $1.40 \cdot 10^{-14}$ & $1.11 \cdot 10^{-16}$ & $2.22 \cdot 10^{-16}$ \\
0.7 & $4.38 \cdot 10^{-14}$ & $2.88 \cdot 10^{-14}$ & $1.82 \cdot 10^{-14}$ & 0 & $3.33 \cdot 10^{-16}$ & 0 \\
0.8 & $5.16 \cdot 10^{-14}$ & $2.29 \cdot 10^{-14}$ & $2.66 \cdot 10^{-15}$ & $1.11 \cdot 10^{-16}$ & $2.22 \cdot 10^{-16}$ & $2.22 \cdot 10^{-16}$ \\
0.9 & $1.21 \cdot 10^{-13}$ & $1.59 \cdot 10^{-13}$ & $1.61 \cdot 10^{-13}$ & 0 & $4.44 \cdot 10^{-16}$ & $2.33 \cdot 10^{-16}$ \\
1.0 & $8.80 \cdot 10^{-12}$ & $1.40 \cdot 10^{-11}$ & $2.05 \cdot 10^{-11}$ & $8.43 \cdot 10^{-15}$ & $3.03 \cdot 10^{-14}$ & $7.43 \cdot 10^{-15}$ \\
\hline
\end{tabular}

method is very convenient for all choices of $\alpha$ and $\beta$ and produces highly accurate solutions to the radial form of the Yamabe equation. Note that since no exact solution exists to (28), residual errors are the best way to deduce the error properties of solutions.

In the case of $\alpha=\beta=1$, the approximate solution by the presented method is shown in Figure $1, \lambda=0.5,0.33,0.25$,
$0.20,0.16, n=3 / 2$, and $\theta=\vartheta=1$ at $N=8$. These plots show that the obtained solutions increase radially from a minimal value which occurs at $r=0$ to a maximal value of 1 which occurs on the boundary.

Logarithmic graphs of absolute coefficients of shifted Jacobi polynomials of (13) are shown in Figures 2 and 3 and show that the method has exponential convergence rate. 
TABLE 4: Absolute residual error functions for $n=3, \lambda=4 / 3$.

\begin{tabular}{lcccccc}
\hline$r$ & $\alpha=\beta=-1 / 2$ & $\begin{array}{l}\alpha=\beta=0 \\
(N=16)\end{array}$ & $\alpha=\beta=1 / 2$ & $\alpha=\beta=-1 / 2$ & $\begin{array}{c}\alpha=\beta=0 \\
(N=24)\end{array}$ \\
\hline 0.0 & $2.48 \cdot 10^{-12}$ & $9.03 \cdot 10^{-12}$ & $2.18 \cdot 10^{-11}$ & $1.21 \cdot 10^{-14}$ & $5.34 \cdot 10^{-15}$ \\
0.1 & $1.85 \cdot 10^{-12}$ & $2.44 \cdot 10^{-12}$ & $2.47 \cdot 10^{-12}$ & $2.22 \cdot 10^{-16}$ & $1.55 \cdot 10^{-15}$ \\
0.2 & $2.15 \cdot 10^{-12}$ & $9.67 \cdot 10^{-13}$ & $1.20 \cdot 10^{-12}$ & $1.77 \cdot 10^{-15}$ & $2.44 \cdot 10^{-15}$ & $1.33 \cdot 10^{-15}$ \\
0.3 & $3.73 \cdot 10^{-12}$ & $2.54 \cdot 10^{-12}$ & $1.66 \cdot 10^{-12}$ & $4.44 \cdot 10^{-16}$ & $6.66 \cdot 10^{-16}$ \\
0.4 & $4.62 \cdot 10^{-12}$ & $3.28 \cdot 10^{-12}$ & $2.34 \cdot 10^{-12}$ & $1.33 \cdot 10^{-15}$ & $2.22 \cdot 10^{-16}$ \\
0.5 & $5.61 \cdot 10^{-12}$ & $4.00 \cdot 10^{-12}$ & $2.90 \cdot 10^{-12}$ & $4.44 \cdot 10^{-16}$ & $2.22 \cdot 10^{-16}$ \\
0.6 & $7.00 \cdot 10^{-12}$ & $4.99 \cdot 10^{-12}$ & $3.57 \cdot 10^{-12}$ & $4.44 \cdot 10^{-16}$ & $1.33 \cdot 10^{-15}$ \\
0.7 & $8.92 \cdot 10^{-12}$ & $6.09 \cdot 10^{-12}$ & $4.01 \cdot 10^{-12}$ & $4.44 \cdot 10^{-16}$ & $1.55 \cdot 10^{-15}$ \\
0.8 & $8.96 \cdot 10^{-12}$ & $4.06 \cdot 10^{-12}$ & $5.12 \cdot 10^{-13}$ & $8.88 \cdot 10^{-16}$ & $4.44 \cdot 10^{-16}$ \\
0.9 & $1.78 \cdot 10^{-11}$ & $2.40 \cdot 10^{-11}$ & $2.50 \cdot 10^{-11}$ & 0 & 0 \\
1.0 & $1.10 \cdot 10^{-9}$ & $1.80 \cdot 10^{-9}$ & $2.69 \cdot 10^{-9}$ & $8.54 \cdot 10^{-15}$ & $8.72 \cdot 10^{-14}$ & $8.88 \cdot 10^{-16}$ \\
\hline
\end{tabular}

TABLE 5: Absolute residual error functions for $n=7 / 3, \lambda=1 / 3$.

\begin{tabular}{|c|c|c|c|c|c|c|}
\hline$r$ & $\alpha=\beta=-1 / 2$ & $\begin{array}{l}\alpha=\beta=0 \\
(N=16)\end{array}$ & $\alpha=\beta=1 / 2$ & $\alpha=\beta=-1 / 2$ & $\begin{array}{l}\alpha=\beta=0 \\
(N=24)\end{array}$ & $\alpha=\beta=1 / 2$ \\
\hline 0.0 & $3.11 \cdot 10^{-15}$ & $8.27 \cdot 10^{-15}$ & $4.01 \cdot 10^{-15}$ & $1.94 \cdot 10^{-14}$ & $4.79 \cdot 10^{-15}$ & $1.09 \cdot 10^{-14}$ \\
\hline 0.1 & $1.11 \cdot 10^{-16}$ & $2.22 \cdot 10^{-16}$ & $1.11 \cdot 10^{-16}$ & $4.44 \cdot 10^{-16}$ & $1.11 \cdot 10^{-16}$ & 0 \\
\hline 0.2 & $1.11 \cdot 10^{-16}$ & $1.11 \cdot 10^{-16}$ & $1.11 \cdot 10^{-16}$ & $1.11 \cdot 10^{-16}$ & $2.22 \cdot 10^{-16}$ & 0 \\
\hline 0.3 & $1.11 \cdot 10^{-16}$ & $1.11 \cdot 10^{-16}$ & $1.11 \cdot 10^{-16}$ & $2.22 \cdot 10^{-16}$ & 0 & $1.11 \cdot 10^{-16}$ \\
\hline 0.4 & $2.22 \cdot 10^{-16}$ & $1.11 \cdot 10^{-16}$ & 0 & 0 & $2.22 \cdot 10^{-16}$ & $2.22 \cdot 10^{-16}$ \\
\hline 0.5 & $1.11 \cdot 10^{-16}$ & $2.22 \cdot 10^{-16}$ & $1.11 \cdot 10^{-16}$ & $1.11 \cdot 10^{-16}$ & $2.22 \cdot 10^{-16}$ & 0 \\
\hline 0.6 & 0 & $1.11 \cdot 10^{-16}$ & $1.11 \cdot 10^{-16}$ & 0 & 0 & $1.11 \cdot 10^{-16}$ \\
\hline 0.7 & $1.11 \cdot 10^{-16}$ & $1.11 \cdot 10^{-16}$ & $3.33 \cdot 10^{-16}$ & $1.11 \cdot 10^{-16}$ & $3.33 \cdot 10^{-16}$ & $1.11 \cdot 10^{-16}$ \\
\hline 0.8 & $1.11 \cdot 10^{-16}$ & $1.11 \cdot 10^{-16}$ & $1.11 \cdot 10^{-16}$ & $1.11 \cdot 10^{-16}$ & $1.11 \cdot 10^{-16}$ & $2.22 \cdot 10^{-16}$ \\
\hline 0.9 & $2.22 \cdot 10^{-16}$ & $2.22 \cdot 10^{-16}$ & $2.22 \cdot 10^{-16}$ & 0 & $1.11 \cdot 10^{-16}$ & $1.11 \cdot 10^{-16}$ \\
\hline 1.0 & $4.88 \cdot 10^{-15}$ & $2.55 \cdot 10^{-15}$ & $1.39 \cdot 10^{-14}$ & $1.36 \cdot 10^{-14}$ & $1.53 \cdot 10^{-14}$ & $2.22 \cdot 10^{-14}$ \\
\hline
\end{tabular}

TABLE 6: Absolute residual error functions for $n=2, \lambda=-1 / 3$.

\begin{tabular}{lcccccc}
\hline$r$ & $\alpha=\beta=-1 / 2$ & $\begin{array}{c}\alpha=\beta=0 \\
(N=16)\end{array}$ & $\alpha=\beta=1 / 2$ & $\alpha=\beta=-1 / 2$ & $\begin{array}{l}\alpha=\beta=0 \\
(N=24)\end{array}$ \\
\hline 0.0 & $1.66 \cdot 10^{-14}$ & $4.83 \cdot 10^{-15}$ & $6.70 \cdot 10^{-15}$ & $1.39 \cdot 10^{-14}$ & $1.49 \cdot 10^{-14}$ \\
0.1 & $7.77 \cdot 10^{-16}$ & $1.11 \cdot 10^{-16}$ & $4.99 \cdot 10^{-16}$ & $2.77 \cdot 10^{-16}$ & $5.55 \cdot 10^{-17}$ & $2.90 \cdot 10^{-14}$ \\
0.2 & $6.10 \cdot 10^{-16}$ & 0 & $1.11 \cdot 10^{-16}$ & 0 & $5.55 \cdot 10^{-17}$ \\
0.3 & $4.44 \cdot 10^{-16}$ & $1.66 \cdot 10^{-16}$ & 0 & $3.88 \cdot 10^{-16}$ & $5.55 \cdot 10^{-17}$ \\
0.4 & $2.22 \cdot 10^{-16}$ & $1.66 \cdot 10^{-16}$ & 0 & $5.55 \cdot 10^{-17}$ & $3.88 \cdot 10^{-16}$ \\
0.5 & $2.77 \cdot 10^{-16}$ & $3.33 \cdot 10^{-16}$ & $2.22 \cdot 10^{-16}$ & 0 & $5.55 \cdot 10^{-17}$ \\
0.6 & $3.88 \cdot 10^{-16}$ & $2.77 \cdot 10^{-16}$ & $1.11 \cdot 10^{-16}$ & 0 & 0 \\
0.7 & $4.44 \cdot 10^{-16}$ & $1.11 \cdot 10^{-16}$ & $3.33 \cdot 10^{-16}$ & $2.22 \cdot 10^{-16}$ & $5.55 \cdot 10^{-17}$ & $1.11 \cdot 10^{-16}$ \\
0.8 & $5.55 \cdot 10^{-16}$ & 0 & $1.11 \cdot 10^{-16}$ & $5.55 \cdot 10^{-16}$ & $2.22 \cdot 10^{-16}$ & $1.66 \cdot 10^{-16}$ \\
0.9 & $1.13 \cdot 10^{-15}$ & $1.11 \cdot 10^{-15}$ & $1.19 \cdot 10^{-15}$ & $3.60 \cdot 10^{-16}$ & $2.22 \cdot 10^{-16}$ \\
1.0 & $6.65 \cdot 10^{-14}$ & $8.56 \cdot 10^{-14}$ & $1.39 \cdot 10^{-13}$ & $2.03 \cdot 10^{-14}$ & $3.58 \cdot 10^{-14}$ & $3.05 \cdot 10^{-16}$ \\
\hline
\end{tabular}

This implies that accurate solutions can be obtained after relatively few iterations of the spectral method (i.e., using relatively few nodes). Therefore, the derived method is quite efficient for use with the Yamabe equation.

Figures 4 and 5 show that the residual error functions for $n=3 / 2$ and $\lambda=0.5,-0.5, N=24, \alpha=\beta=1$. The errors are absolute, as opposed to relative. Again, there are no exact solutions to problems (1)-(2), we must rely on residual errors rather than any exact errors or errors relative to an exact solution. We see that the residual errors are extremely small on the problem domain, owing to the the accuracy of the method.

With this, we have demonstrated the accuracy and efficiency of the proposed spectral method for radial Yamabe 


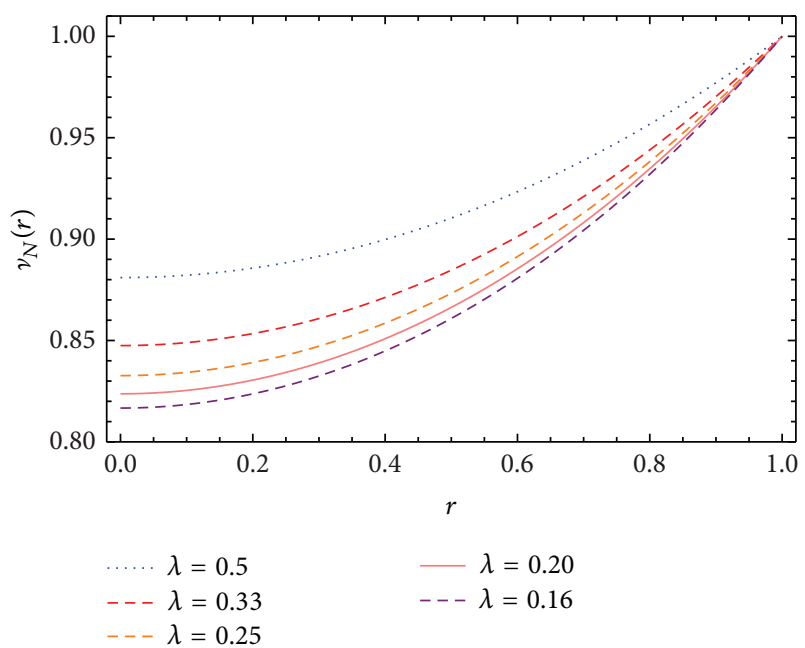

FIGURE 1: Plots of the solution $v(r)$ for the radial Yamabe equation obtained by the present method for $\lambda=0.5,0.33,0.25,0.20,0.16$, $n=3 / 2$, and $\theta=9=1$ at $N=8$.

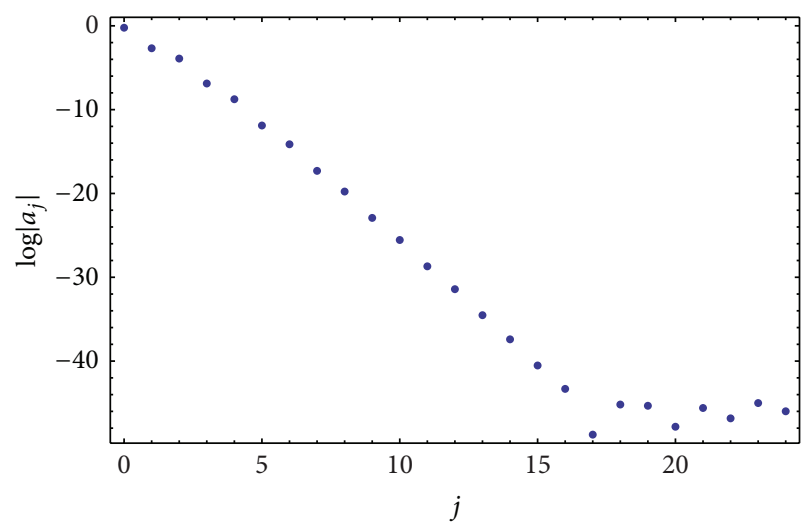

FigURE 2: Logarithmic graph of absolute coefficients $\left|a_{j}\right|$ of shifted Jacobi polynomials for $n=3 / 2, \lambda=-0.5$, and $\alpha=\beta=1$ at $N=24$.

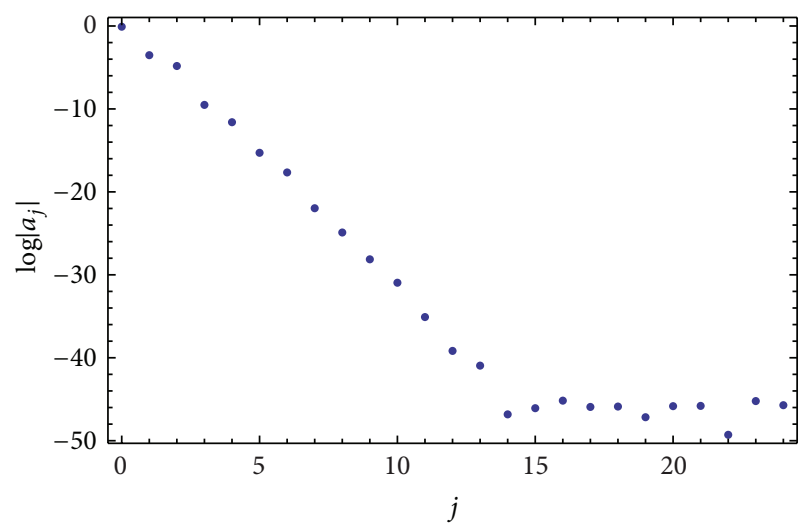

FIGURE 3: Logarithmic graph of absolute coefficients $\left|a_{j}\right|$ of shifted Jacobi polynomials for $n=3 / 2, \lambda=0.5$, and $\alpha=\beta=1$ at $N=24$.

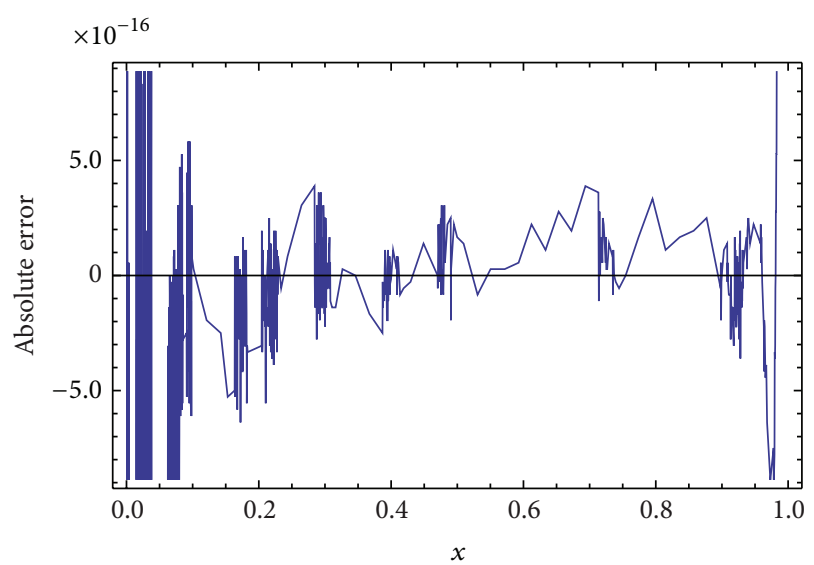

FIGURE 4: Graph of residual error functions for $n=3 / 2, \lambda=-0.5$, and $\alpha=\beta=1$ at $N=24$.

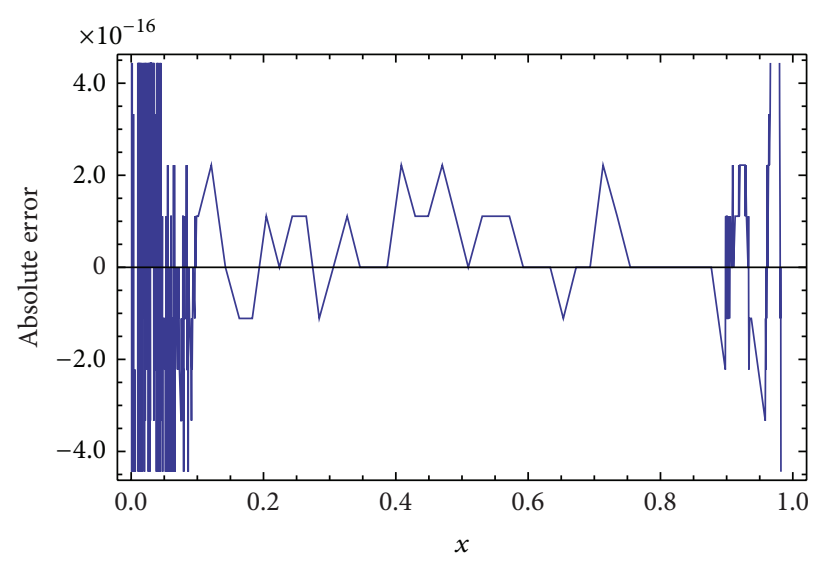

FIGURE 5: Graph of residual error functions for $n=3 / 2, \lambda=0.5$, and $\alpha=\beta=1$ at $N=24$.

equation (28), for a number of model parameters. Importantly, the method remains applicable for a variety of values of $n$, which directly controls the extent of the nonlinearity in the problem. Therefore, the method appears rather robust in light of the strong nonlinearity inherent in the Yamabe problem.

\section{Conclusions}

We have derived a spectral method, involving Jacobi polynomials, which permits us to numerically solve a class of nonlinear boundary value problems which feature powerlaw nonlinearity. For suitable collocation points, we use the $N-1$ nodes of the shifted Jacobi-Gauss interpolation on $(0,1)$. These equations together with initial condition generate $(N+1)$ algebraic equations which can be solved using Newton's iterative method. For the parameter regimes considered, the coefficients of the shifted Jacobi polynomials in the solutions of the boundary value problem are shown to decay exponentially in magnitude. This implies that the method has a rapid convergence rate, which in turn tells us that accurate solutions can be obtained after relatively 
few iterations of the spectral method (i.e., using relatively few nodes). In other words, the method is rather efficient for solving the class of nonlinear boundary value problems considered.

Indeed, in Section 4, we demonstrated that for all parameter regimes considered, the method results in rather small residual errors after relatively few nodes are considered. As such, we may infer that the method is accurate in approximating a true solution to boundary value problems of the type considered here, such as that for the radial Yamabe equation. We have considered a number of forms of nonlinearity in our numerical experiments, corresponding to different values of the power index $n$ which governed the strength of the nonlinearity. Therefore, the spectral method is accurate and efficient for a wide variety of nonlinearities. In the case of the Yamabe problem, mapping the solutions back to the original $m$-dimensional space, we see that the method is robust for arbitrary space dimension $m \geq 3$. Recall that the parameter $n$ is a decreasing function of $m$, and the strongest nonlinearity actually occurs for $m=3$ in the Yamabe example.

Since the method is rather robust, it is likely that it may be applied to other type of non-linear boundary value problems with more complicated forms of nonlinearity. For instance, based on the results here, it seems likely that the method derived in Section 3 can be extended to problems of the type

$$
\begin{gathered}
y^{\prime \prime}(r)+\frac{1}{r} y^{\prime}(r)=P(y(r)), \\
y^{\prime}(0)=0, \quad y(1)=1 .
\end{gathered}
$$

\section{Conflict of Interests}

The authors declare that there is no conflict of interests regarding the publication of this paper.

\section{Acknowledgments}

This paper was funded by the Deanship of Scientific Research DSR, King Abdulaziz University, Jeddah. The authors, therefore, acknowledge with thanks DSR technical and financial support. R.A.V. supported in part by NSF Grant no. 1144246 .

\section{References}

[1] C. Canuto, M. Y. Hussaini, A. Quarteroni, and T. A. Zang, Spectral Methods in Fluid Dynamics, Springer, New York, NY, USA, 1988.

[2] B. Fornberg, A Practical Guide to Pseudospectral Methods, vol. 1, Cambridge University Press, Cambridge, UK, 1998.

[3] E. H. Doha, A. H. Bhrawy, D. Baleanu, and R. M. Hafez, "A new Jacobi rational-Gauss collocation method for numerical solution of generalized pantograph equations," Applied Numerical Mathematics., vol. 77, pp. 43-54, 2014.

[4] R. Peyret, Spectral Methods for Incompressible Viscous Flow, vol. 148, Springer, New York, NY, USA, 2002.

[5] L. N. Trefethen, Spectral Methods in MATLAB, vol. 10, SIAM, Philadelphia, Pa, USA, 2000.
[6] E. H. Doha, A. H. Bhrawy, D. Baleanu, and M. A. Abdelkawy, "Numerical treatment of coupled nonlinear hyperbolic KleinGordon equations," Romanian Journal of Physics, vol. 59, pp. 247-264, 2014.

[7] F. Yin, J. Song, Y. Wu, and L. Zhang, "Numerical solution of the fractional partial differential equations by the twodimensional fractional-order Legendre functions," Abstract and Applied Analysis, vol. 2013, Article ID 562140, 13 pages, 2013.

[8] D. Baleanu, A. H. Bhrawy, and T. M. Taha, "Two efficient generalized Laguerre spectral algorithms for fractional initial value problems," Abstract and Applied Analysis, vol. 2013, Article ID 546502, 10 pages, 2013.

[9] E. H. Doha, A. H. Bhrawy, R. M. Hafez, and R. A. van Gorder, "A Jacobi rational pseudospectral method for Lane-Emden initial value problems arising in astrophysics on a semi-infinite interval," Computational and Applied Mathematics, 2013.

[10] R. C. Mittal and R. Bhatia, "Numerical solution of second order one dimensional hyperbolic telegraph equation by cubic Bspline collocation method," Applied Mathematics and Computation, vol. 220, pp. 496-506, 2013.

[11] Y. Yang and Y. Huang, "Spectral-collocation methods for fractional pantograph delay-integrodifferential equations," Advances in Mathematical Physics, vol. 2013, Article ID 821327, 14 pages, 2013.

[12] M. H. Heydari, M. R. Hooshmandasl, C. Cattani, and M. Li, "Legendre wavelets method for solving fractional population growth model in a closed system," Mathematical Problems in Engineering, vol. 2013, Article ID 161030, 8 pages, 2013.

[13] A. H. Bhrawy and M. A. Alghamdi, "A shifted Jacobi-GaussLobatto collocation method for solving nonlinear fractional Langevin equation involving two fractional orders in different intervals," Boundary Value Problems, vol. 2012, p. 62, 2012.

[14] E. H. Doha, A. H. Bhrawy, and W. M. Abd-Elhameed, "Jacobi spectral Galerkin method for elliptic Neumann problems," Numerical Algorithms, vol. 50, no. 1, pp. 67-91, 2009.

[15] A. H. Bhrawy, "A Jacobi-Gauss-Lobatto collocation method for solving generalized Fitzhugh-Nagumo equation with timedependent coefficients," Applied Mathematics and Computation, vol. 222, pp. 255-264, 2013.

[16] E. H. Doha, A. H. Bhrawy, M. A. Abdelkawy, and R. A. van Gorder, "Jacobi-Gauss-Lobatto collocation method for the numerical solution of $1+1$ nonlinear Schrödinger equations," Journal of Computational Physics, vol. 261, pp. 244-255, 2014.

[17] W. Heinrichs, "Spectral approximation of third-order problems," Journal of Scientific Computing, vol. 14, no. 3, pp. 275-289, 1999.

[18] K. Aghigh, M. Masjed-Jamei, and M. Dehghan, "A survey on third and fourth kind of Chebyshev polynomials and their applications," Applied Mathematics and Computation, vol. 199, no. 1, pp. 2-12, 2008.

[19] M. Fernandino, C. A. Dorao, and H. A. Jakobsen, "Jacobi Galerkin spectral method for cylindrical and spherical geometries," Chemical Engineering Science, vol. 62, pp. 6777-6783, 2007.

[20] E. H. Doha and A. H. Bhrawy, "An efficient direct solver for multidimensional elliptic Robin boundary value problems using a Legendre spectral-Galerkin method," Computers \& Mathematics with Applications, vol. 64, no. 4, pp. 558-571, 2012.

[21] E. H. Doha, A. H. Bhrawy, and R. M. Hafez, "On shifted Jacobi spectral method for high-order multi-point boundary value problems," Communications in Nonlinear Science and Numerical Simulation, vol. 17, no. 10, pp. 3802-3810, 2012. 
[22] S. Y. Alice Chang, Z. C. Han, and P. Yang, "Classification of singular radial solutions to the [sigma] $\mathrm{k}$ Yamabe equation on annular domains," Journal of Differential Equations, vol. 216, pp. 482-501, 2005.

[23] L. Andersson, P. T. Chruściel, and H. Friedrich, "On the regularity of solutions to the Yamabe equation and the existence of smooth hyperboloidal initial data for Einstein's field equations," Communications in Mathematical Physics, vol. 149, no. 3, pp. 587-612, 1992.

[24] S. Brendle, "Blow-up phenomena for the Yamabe equation," Journal of the American Mathematical Society, vol. 21, no. 4, pp. 951-979, 2008.

[25] R. A. van Gorder, "Control of error in the homotopy analysis of semi-linear elliptic boundary value problems," Numerical Algorithms, vol. 61, no. 4, pp. 613-629, 2012.

[26] H. Adibi and A. M. Rismani, "On using a modified Legendrespectral method for solving singular IVPs of Lane-Emden type," Computers \& Mathematics with Applications, vol. 60, no. 7, pp. 2126-2130, 2010.

[27] A. H. Bhrawy and A. S. Alofi, "A Jacobi-Gauss collocation method for solving nonlinear Lane-Emden type equations," Communications in Nonlinear Science and Numerical Simulation, vol. 17, no. 1, pp. 62-70, 2012.

[28] K. Parand, M. Shahini, and M. Dehghan, "Rational Legendre pseudospectral approach for solving nonlinear differential equations of Lane-Emden type," Journal of Computational Physics, vol. 228, no. 23, pp. 8830-8840, 2009.

[29] R. A. van Gorder and K. Vajravelu, "Analytic and numerical solutions to the Lane-Emden equation," Physics Letters A, vol. 372, no. 39, pp. 6060-6065, 2008. 


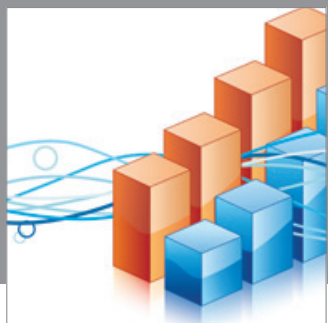

Advances in

Operations Research

mansans

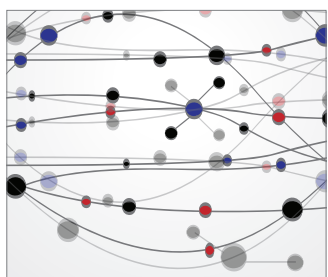

The Scientific World Journal
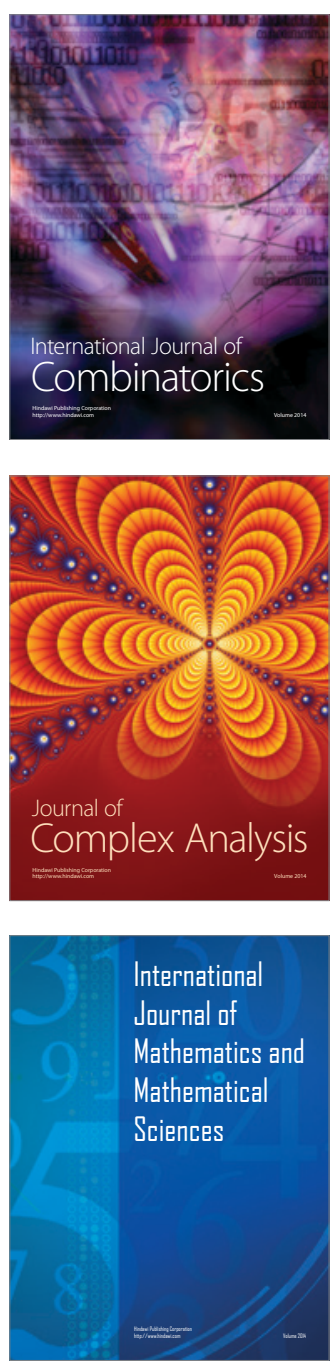
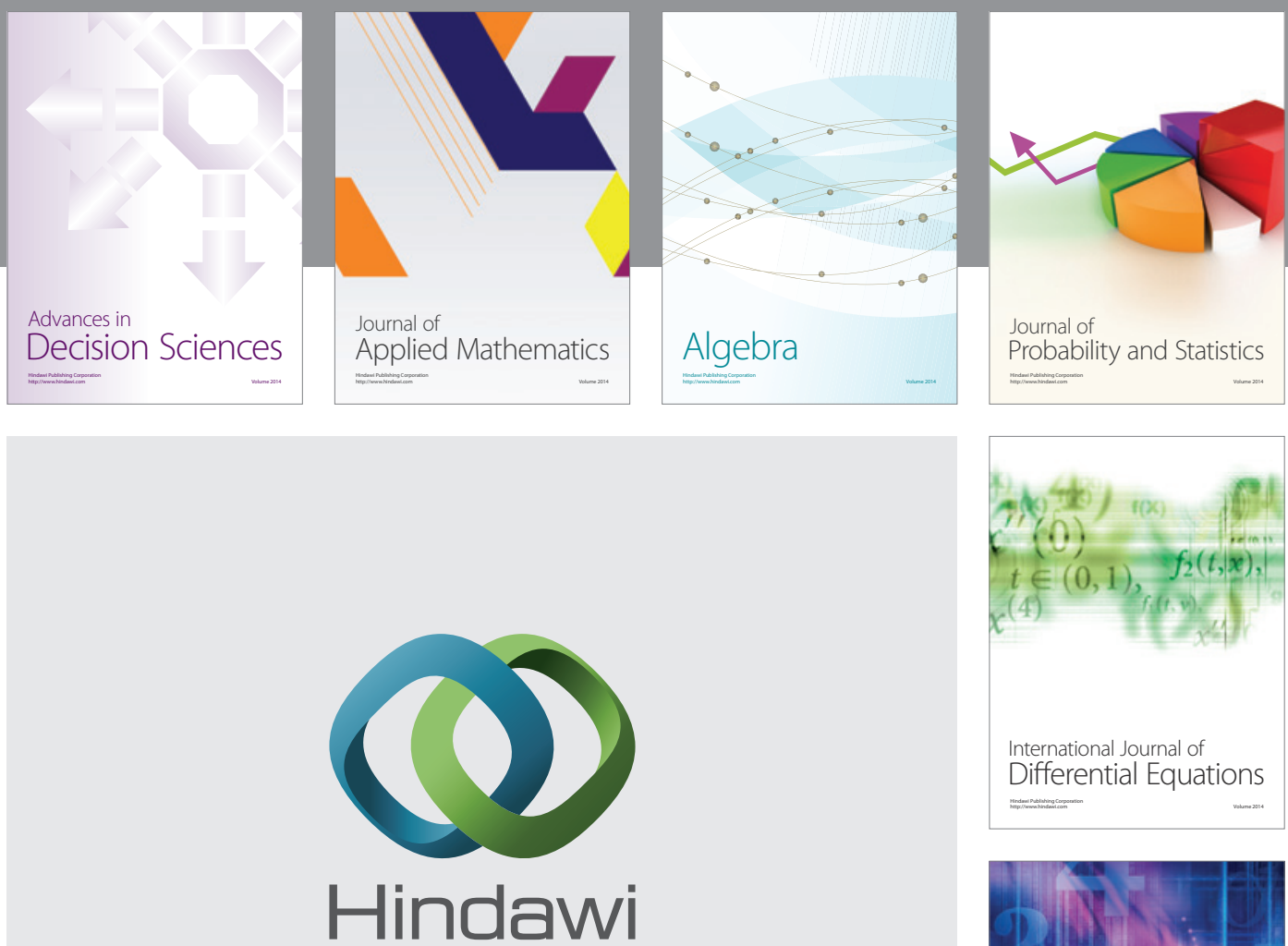

Submit your manuscripts at http://www.hindawi.com
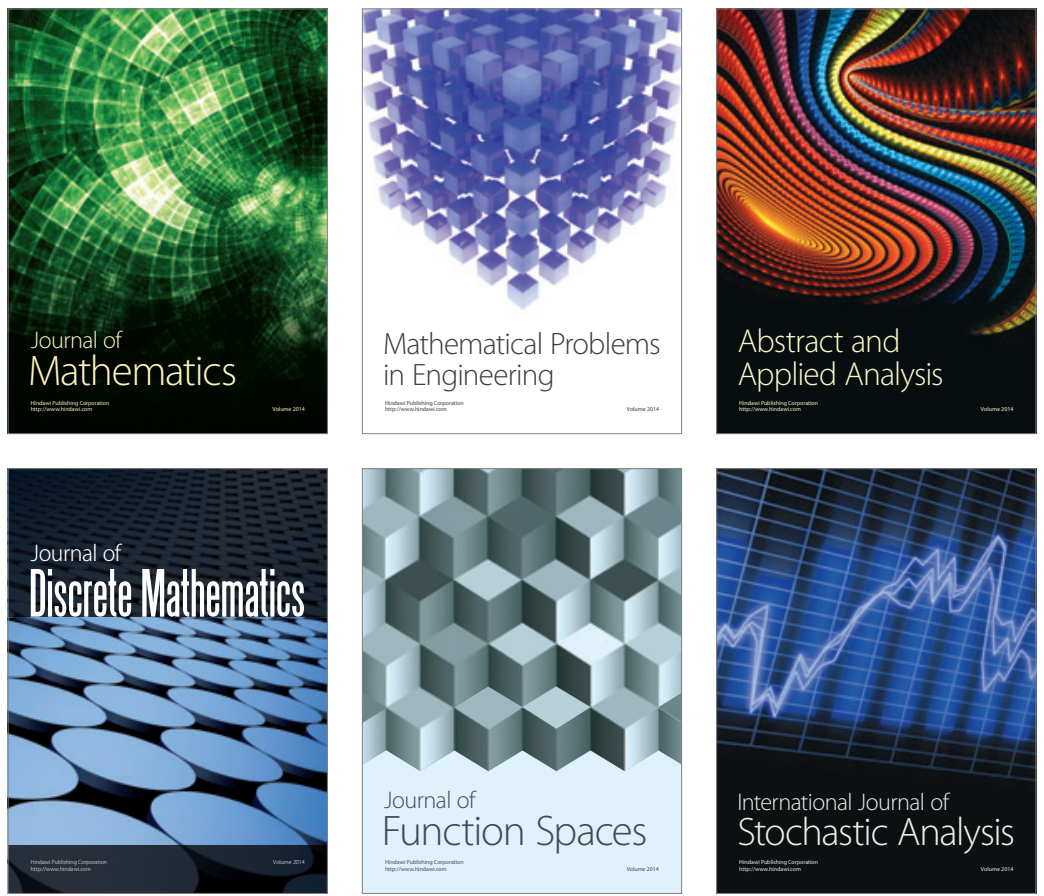

Journal of

Function Spaces

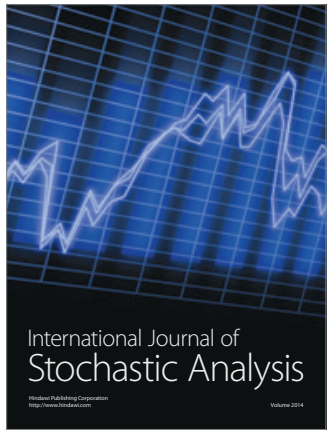

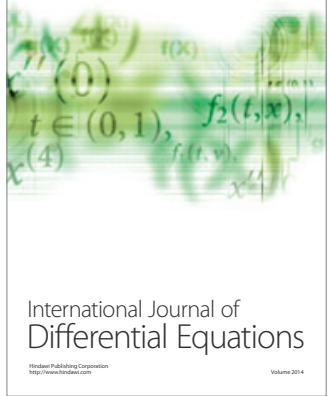
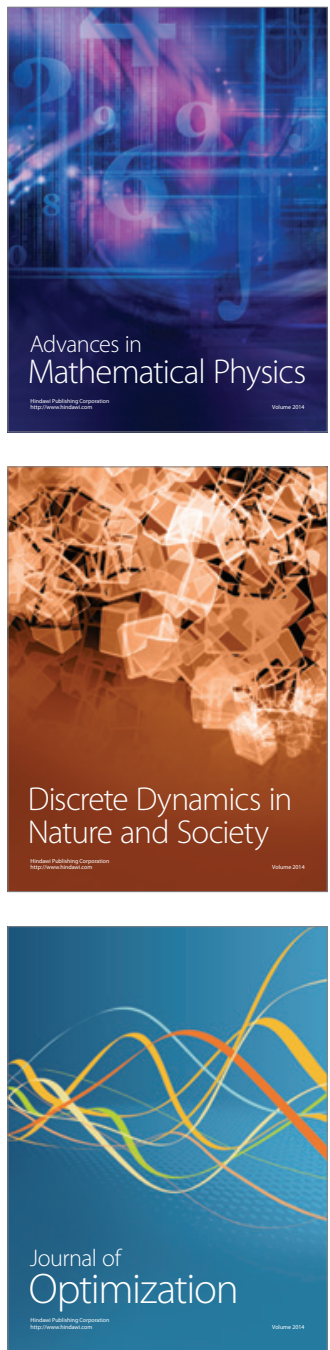